\title{
POURQUOI ET COMMENT CHERCHER A OBTENIR DES NAISSANCES GÉMELLAIRES CHEZ LES BOVINS
}

\author{
I. -- MO'TIVATIONS POUR UN PROGRAMME DE RECHERCHE \\ SUR IES NAISSANCES GÉMELI,AIRES
}

Le problème de l'équilibre lait/viande se posait déjà avec une certaine acuité lorsque la Délégation Générale à la Recherche Scientifique et Technique décida en I963 d'aider 1'Institut National de la Recherche Agronomique au démarrage d'études importantes sur les naissances gémellaires. Par la suite ce problème n'a fait que s'amplifier. Les quantités de lait livrées à l'industrie laitière ont augmenté régulièrement par suite de l'amélioration rapide des techniques de production. De I962 à I967 la production de lait de vache a progressé de ro,9 p. Ioo dans la Communauté Économique Européenne et de 20,8 p. roo en France. La consommation ne s'est pas accrue avec la même intensité et les stocks de beurre de la Communauté qui atteignaient à peine Ioo ooo tonnes en I962 dépassaient 200 ooo tonnes en I967 et représentaient près de la moitié du stock mondial. Étant donné la situation du marché mondial des produits laitiers, l'écoulement des excédents sur les marchés extérieurs de la Communauté ne peut se faire qu'à des prix inférieurs à ceux du Marché Commun.

Au contraire la consommation de viande bovine augmente plus rapidement que la production, d'où l'existence d'un déficit permanent de la Communauté qui oscille autour de 500 ooo tonnes annuellement. "Ainsi la Communauté se ruine deux fois : une première fois pour vendre son beurre, une deuxième fois pour acheter son bifteck. Six millions de prélèvement sur ses ressources, tel est le prix actuel du rapport lait/viande " (PÉro, Ig68).

L'excédent de viande bovine exporté par la France est insuffisant pour rétablir la situation puisqu'il oscille autour de Ioo ooo tonnes seulement. Cette situation pourrait offrir théoriquement une occasion d'expansion aux races à viande. Cependant celles-ci ne peuvent se développer que si le produit brut annuel par vache est augmenté. Les essais d'introduction de telles races en Suède ont bien montré cette difficulté (PORIN, I968). Une étude précise effectuée par BILLÈrE (Ig66) sur la race Charolaise a clairement établi que ce produit brut était en grande partie lié au nombre de veaux qui peuvent être produits annuellement par un troupeau de vaches. Cet 
auteur a trouvé que le taux de fécondité (nombre de veaux sevrés pour Ioo vaches mises à la reproduction) était en moyenne de 78,4 p. Ioo ; ce chiffre rejoint les estimations du Département de 1'Agriculture américain pour lequel la récolte de veaux pour l'ensemble du bétail à viande des États-Unis a été de 79,5 p. Ioo en I967. BILLìne (I966) a calculé que le prix de revient du veau sevré diminuerait de près de $20 \mathrm{p}$. Ioo si le taux de fécondité passait à $95 \mathrm{p}$. Ioo et de $50 \mathrm{p}$. Ioo si ce taux était de 550 p. roo. Même sans atteindre ce dernier chiffre, la situation économique des races à viande serait très améliorée si on obtenait un taux de fécondité de I20-I 25 p. IOO. Ce résultat ne peut être obtenu, dans 1'état actuel des choses, qu'en augmentant la fréquence des naissances gémellaires. Ce faisant on améliorerait aussi les conditions de vêlage souvent difficiles de certaines races à vianđe puisque les veaux jumeaux sont plus petits que les simples. Pour la race Charolaise, Bil signale que $6,8 \mathrm{p}$. Ioo des veaux et $\mathrm{r}, 3 \mathrm{p}$. Ioo des vaches sont perdus à la mise bas essentiellement à la suite de vêlages dystociques. De même VANDEnbussche et al. (I964) rapportent que pour la race Ronge des Flandres, 5,8 p. Ioo des veaux sont extraits par césarienne et I,6 p. Ioo par embryotomie par suite d'une trop forte taille.

Parallèlement on peut envisager des ateliers de vaches de races spécialisées pour la production laitière participant plus fortement à la production de viande par des naissances gémellaires de veaux issus de croisement avec des taureaux de races à viande. On diminuerait aussi la quantité de lait commercialisé par vache.

Ainsi s'impose la nécessité de mise au point d'une technique permettant l'obtention de naissances gémellaires chez les Bovins.

\section{II. - NAISSANCES GÉMELLAIRES NORMALEMENT OBSERVÉES DANS LES TROUPEAUX BOVINS}

Les naissances gémellaires sont en effet normalement observées dans l'espèce bovine. Leur taux d'apparition varie selon les races de 0,4 p. Ioo à 4,6 p. Ioo (tabl. I). Les fréquences les plus élevées parmi les races laitières sont observées dans les races Simmenthal, Rouge des Steppes et Holstein-Friesian et dans la race Charolaise pour les races à viande. Les fréquences les plus basses semblent avoir été détectées dans les races Limousine, Hereford et Aberdeen-Angus.

A l'intérieur d'une race, certaines familles montrent une tendance à donner une plus forte proportion de naissances gémellaires (LUSH, I925). Effectivement, les Carnation par exemple, ont un taux de naissances gémellaires plus élevé (4,6 p. IOO) que la moyenne de la race Holstein-Friesian (3,6 p. IOO) (ERB et MORRISON, I959). Il en est de même de certaines lignées de Charolaise (VISSAC, communication personnelle).

L'âge de la mère constitue l'un des facteurs de variation les plus importants. En général, la proportion des naissances gémellaires est très faible au premier vêlage (o à I,3 p. IOO), elle augmente brutalement ( 2 à 3 fois) au deuxième vêlage, puis tend à s'accroître assez régulièrement au cours des vêlages suivants, au moins jusqu'au huitième ou neuvième pour atteindre un maximum de 2,5 à $9 \mathrm{p}$. Ioo selon les races 
(Hewitt, I934 ; Pfau et al., I948 ; Erb et Morrison, I959 ; Boyd et REEd, I96I ; Brodauf, I963; Maijala, I964).

Bien que les naissances gémellaires apparaissent toute l'année, leur fréquence montre une certaine tendance à des variations saisonnières. Les fréquences les plus élevées résultent de conceptions ayant eu lieu à l'automne et au début de l'hiver (BRODAUF, I963), ou lors de la mise à l'herbe (FREY, I959; VISSAC, communication personnelle). Il existerait en outre des variations importantes d'une année sur l'autre (MAIJAI, I964).

TABLEAU I

Fréquences de naissances multiples normalement observées

\begin{tabular}{|c|c|c|}
\hline & Fréquence $(\%)$ & Référence \\
\hline \multicolumn{3}{|l|}{ Races laitières } \\
\hline 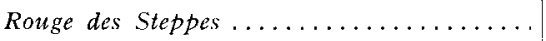 & 4,36 & MaRtinenko $(1965)$ \\
\hline 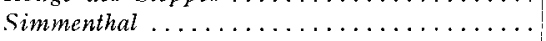 & 4,61 & JohansSON et VENGE (1951) \\
\hline Simmenthal $\ldots \ldots \ldots \ldots \ldots \ldots \ldots$ & 3,69 & RICHTER (1955) \\
\hline 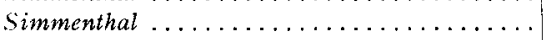 & 3,06 & Martinenko $(1965)$ \\
\hline Simmenthal $\ldots \ldots \ldots \ldots \ldots \ldots \ldots \ldots \ldots$ & 2,37 & JiGLE $(1964)$ \\
\hline 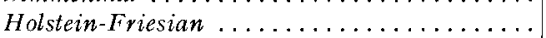 & 4,16 & PFAU et al. $(1918)$ \\
\hline 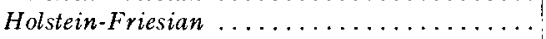 & 3,08 & Meadows et Lush (1957) \\
\hline 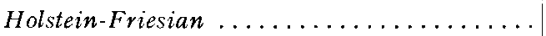 & 3,57 & ERB et Morrison $(1959)$ \\
\hline Frisonne Suédoise .................. & 3,32 & JohansSon et VENGE $(1951)$ \\
\hline Frisonne australienne .............. & 3,06 & HewrtT (1934) \\
\hline 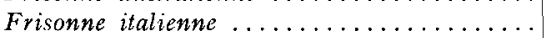 & 3,10 & Matassino et Gargiclo $(1965)$ \\
\hline Frisonne britannique $\ldots \ldots \ldots \ldots \ldots \ldots$ & 2,70 & Boyd et REED (1961) \\
\hline 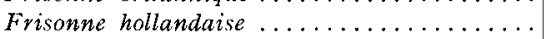 & 1,30 & DABASH $(1964)$ \\
\hline Frisonne d'Afrique du Sud .............. & 1,30 & JoUBERT (1952) \\
\hline 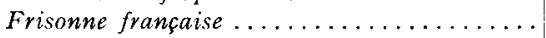 & 1,10 & MENISSILR $(1965)$ \\
\hline Ayrshive $\ldots \ldots \ldots \ldots \ldots \ldots \ldots \ldots$ & 2,81 & MEADOWs et LuSH (1957) \\
\hline 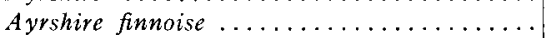 & 1,31 & JoHANSSON et VENGE (1951) \\
\hline 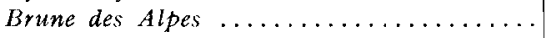 & 2,73 & JOHANSSON et VENGE (1951) \\
\hline Brune de Wurtemberg ............... & 1,94 & FREY $(1959)$ \\
\hline 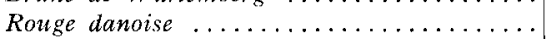 & 2,73 & JohansSon et VENGE (1951) \\
\hline Salers $\ldots \ldots \ldots \ldots \ldots \ldots \ldots \ldots \ldots \ldots$ & 2,53 & Menissier $(1965)$ \\
\hline Normande $\ldots \ldots \ldots \ldots \ldots \ldots \ldots$ & 1,87 & Menissier $(1965)$ \\
\hline Guernesey $\ldots \ldots \ldots \ldots \ldots \ldots \ldots \ldots$ & 1,95 & Meadows et Lush (1957) \\
\hline 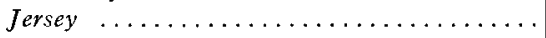 & 1,31 & Meadows et Lush (1957) \\
\hline Jersey $\ldots \ldots \ldots \ldots \ldots \ldots \ldots \ldots \ldots$ & 1,02 & JoHANSSON et VENGE (1951) \\
\hline 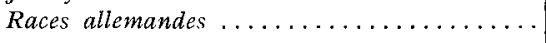 & 1,33 & BRODAUF $(196: 3)$ \\
\hline Races à viande & & \\
\hline 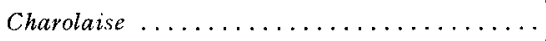 & $2,8-3,1$ & AURIOL et al. (1961) \\
\hline Charolaise $\ldots \ldots \ldots \ldots \ldots \ldots \ldots \ldots \ldots$ & 2,6 & BiLlère (1965) \\
\hline 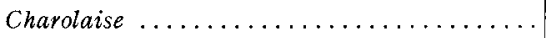 & 2,5 & Menissier $(1965)$ \\
\hline 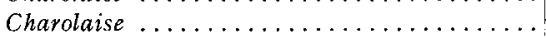 & 3,25 & VISSAC communication personnelie \\
\hline Limousine $\ldots \ldots \ldots \ldots \ldots \ldots \ldots \ldots \ldots$ & 0,57 & MENISSIER (1965) \\
\hline Hereford et Aberdeen-Angus ........... & 0,10 & TURMAN et al. (1968) \\
\hline
\end{tabular}

Ces naissances gémellaires sont en général constituées par des doubles. On constate également l'existence de triplets : 0,OI à o,2 p. IOo (PFAU et al., I948 ; RICHTER, I955) et l'apparition de quadruplets est extrêmement rare : 0,002 p. IOO (RICH'TER, I955). La proportion de vrais jumeaux est faible, 4 à 7 p. roo de l'ensemble des jumeaux 
(Johansson et Vengr, I95I ; MEadows et Lush, I957; ERb et Morrison, I959). La majeure partie des naissances gémellaires résulte donc d'ovulations multiples. KIDDER ct al. (I952) avaient rapporté que la proportion de ces ovulations était égale à I3, I p. IOo chez des Holstein-Friesian; LABHSETWAR et al. (I963), après examen minutieux, ont estimé ce taux à 2,9 p. Ioo chez les nullipares, à 3,7 p. Ioo chez les primipares et à 5,9 p. Ioo chez les multipares. On est donc amené à soupçonner l'existence soit d'une mauvaise fécondabilité des œufs, soit d'une mortalité embryonnaire puisque le taux de naissances gémellaires est inférieur à celui des ovulations multiples.

\section{III. - PROBLÈMES ZOOTECHNIQUES POSÉS PAR LES NAISSANCES GÉMELLAIRES}

L'apparition normale de naissances gémellaires dans les troupeaux bovins laitiers a mis en évidence quelques problèmes zootechniques et soulevé certaines controverses.

Ainsi plusieurs auteurs rapportent une augmentation de la fréquence des rétentions placentaires parallèlement à celle des naissances gémellaires (PFAU et al., I948 ; Morrison et Firb, I957; Comberg et Velten, I962; Brands, I966). Le sexe des fœtus semble d'ailleurs influencer la proportion des rétentions placentaires : celle-ci serait plus élevée dans le cas de veaux mâles (COMBERG et VEITEN, I962). I1 est cependant difficile de comparer les différents résultats car les intervalles après parturition varient selon les auteurs ; on pourrait admettre cependant que l'augmentation de la fréquence des rétentions placentaires est liée à l'accroissement du nombre de placentomes observés dans le cas de naissances gémellaires (TESTART et I)U MESNIL DU Buisson, Ig66).

Les conséquences des rétentions placentaires sur la vie sexuelle ultérieure de la mère ne sont pas nettes. Elles résultent en général soit d'une infection consécutive à cette situation soit d'une involution insuffisante de l'utérus au moment de l'insémination. Certains auteurs estiment que les vaches ayant donné des naissances gémellaires exigent plus de saillies par conception que les autres vaches (PFAU et al., I948 ; Croninger et Thoele, i957 ; Boyd et Reed, ig6i ; ANonyme, ig66). Par contre ERB et Morrison (I959), COMBERG et VEITEN (I962) ne constatent aucune différence pour ce paramètre entre ces différentes catégories de vaches. Il en résulte que les intervalles entre vêlages sont (PFAU et al., I948 ; IABASH, I964) ou non (CoMBERG et VELTEN, I962) significativement modifiés. EGLE (I964) rapporte que les naissances gémellaires n'affectent pas la fertilité des mères après une étude portant sur 5208 vaches Simmenthal; alors que d'autres auteurs ont une opinion inverse (Brodauf, I963; Cloninger et Thoeis, I957).

La durée de gestation est en général plus courte de 7 à Io jours lorsqu'il s'agit de gestations multifœtales que pour des gestations simples (HEwITT, I934; PFAU et al., I948; FREY, I959; MATASSINO et BORDI, I967). La proportion d'avortements est d'ailleurs légèrement plus élevée dans le cas de gestations multifœtales. Le raccourcissement de la durée de gestation est partiellement responsable du plus faible poids vif à la naissance des jumeaux et des triplets puisque ceux-ci naissent plus 
jeunes que les simples (Johansson, I932; Hewi't', I934; Matassino et Marati, ig64; Petcu et Calotoru, I967). Cependant le poids total des jumeaux excède celui des simples de 36 à 56 p. Ioo ; l'influence du taureau sur le poids des veaux à la naissance est d'ailleurs très élevée dans le cas de jumeaux (DABAsh, I964).

En général le taux de mortalité des veaux à la naissance est plus élevé pour les jumeaux que pour les simples (PFAU et al., I948; ERB et MORrison, I959 ; ComBERG et VELTEN, I962; BRODAUF, I963; DABASH, I964) bien que, dans quelques cas aucune différence n'ait été observée (HEwITT, I934).

La vitesse de croissance des jumeaux est identique ou supérieure à celle des simples (KRIVINSKII, I962; HRISTOV, Ig66) et la production laitière de vaches nées jumelles est très voisine de celle de vaches nées simples (HEwI'Tr, I934; ArzumanJAN, I965; SCHULTZE, I966). Il faut noter cependant que les rendements laitiers des mères donnant naissance à des jumeaux sont en général plus élevés que ceux des vaches donnant naissance à des simples (HEwITT, I934; DABAsh, I964; MEADows et IUSH, I957).

Toutes les jumelles ne sont pas aptes à la reproduction; on a constaté en particulier que $90 \mathrm{p}$. Ioo des femelles, co-jumelles de mâles, sont stériles ou subfertiles: ce sont les free-martins (LILLIE, I9I6, I9I7; KELLER et TANDLER, I9I6). Ce fait est lié à l'établissement de connexions vasculaires entre les deux fœetus jumeaux. RENDEI, (I963) n'a trouvé que 7 p. Ioo de paires de jumeaux dizygotes chez lesquels aucune connexion vasculaire ne semblait avoir existé. Les femelles free-martin peuvent être détectées dès les premières semaines de leur vie puisque la longueur de leur vagin est égale au tiers de celle du vagin des femelles normales.

Ainsi l'étude de la bibliographie révèle l'existence de résultats contradictoires pour la plupart des paramètres zootechniques envisagés. En réalité la majeure partie des observations rapportées ont été effectuées dans des conditions d'élevage et d'hygiène vétérinaire très différentes. On peut donc espérer raisonnablement que les progrès de la médecine vétérinaire et de la zootechnie permettront de surmonter la majorité de ces problèmes. Aussi ne semble-t-il pas utopique d'entreprendre des recherches devant aboutir à 1'augmentation de la fréquence des naissances gémellaires en vue d'une augmentation de la production de la viande bovine.

\section{IV. - ÉTABLISSEMEN'T D'UN PROGRAMME POUR L'OBTENTION EXPÉRIMENTAI, DE NAISSANCES GÉMEI,I,AIRES}

Bien que certaines familles montrent une tendance à une forte proportion d'ovulations (KIDDER et al., I952) ou de naissances gémellaires (L,USH, I925; HewITT, I934; ERB et Morrison, I959; VISSAC, communication personnelle), une tentative de sélection pendant 25 ans sur le taux de naissances gémellaires dans un troupeau Angus n'a donné que peu ou pas de résultats (MEChling et CARTER, I964). De toute façon, la solution génétique à ce problème ne peut être envisagée qu'à longue échéance. C'est pourquoi la solution qui a été mise à l'étude réside dans une stimulation hormonale. Cette voie a déjà été utilisée pour l'obtention des naissances gémellaires par ARBEITER (Ig62), Gordon et al. (rg62) sous 1'égide du Milk Marke- 
ting Board, puis par Martinenko (I965 b) en U. R. S. S., Hafez et al. (I965) et Turman et al. (Ig69) aux U. S. A.

L'obtention de naissances gémellaires par voie hormonale pose cependant de nombreux problèmes. Elle exige en effet que :

- plusieurs ovulations soient induites, ce qui nécessite une stimulation folliculaire préalable par une hormone gonadotrope ;

- les oufs ainsi obtenus soient fécondables et que, malgré l'existence d'une mortalité embryonnaire précoce, un nombre suffisant de blastocystes puissent s'implanter ;

- les embryons implantés se développent normalement et donnent naissance à de jeunes veaux viables.

\section{A. - Nature, dose et moment d'injection des hormones gonadotropes utilisées}

Depuis les travaux de ZAVADowski et Eskin (I939), de nombreux chercheurs ont utilisé des préparations d'hormones gonadotropes pour induire une superovulation sur des vaches sexuellement mûres (CASIDA et al., I943; Hammond et BhatTACHARYA, I944 ; WILLE'T' et al., I953 ; UMBAUGH, I949 ; DzIUK et al., I958 ; HAFEZ et al., I963; HAFEZ et al., I965).

Les préparations d'hormones hypophysaires ont donné en général des résultats prometteurs ; cependant, de telles hormones sont actuellement chères et en quantité limitée. Aussi utilise-t-on généralement 1'hormone extraite du sérum de jument gravide $\left(7 \mathrm{O}^{\mathrm{e}}-\mathrm{I} 2 \mathrm{O}^{\mathrm{e}}\right.$ jours de la gestation), PMSG, pour induire une forte stimulation folliculaire. Le fait que cette hormone possède une demi-vie supérieure à celle des autres hormones gonadotropes représente une propriété intéressante pour l'obtention d'une superovulation (PARLOW, I960). Cependant, cette hormone peut, au cours de traitements répétés, induire la formation d'antihormones qui limitent l'effet de l'hormone elle-même (Core et al., I957 ; NAKAHARA et al., I964; JAINUDEEN et al., I966).

Bien que non indispensable, l'utilisation d'hormone à activité ovulante comme l'hormone lutéinisante $\mathrm{L}_{\mathrm{H}} \mathrm{H}$ ou beaucoup plus fréquemment 1'hormone chorionique humaine permet d'améliorer la proportion de follicules stimulés qui ovulent (SUGrE et al., I96I ; HAFEz et al., I963).

Dans tous les travaux rapportés ici, nous avons utilisé PMSG et HCG et nous avons successivement recherché :

- les doses d'hormones qui permettent d'obtenir une superovulation modérée. En effet, seuls Hammond et Bhattacharya (I944), Hammond Jr. (I949), Gordon et al. (I962), Schilling et Holm (I963), HaFEz et al. (I964) ont essayé d'obtenir une réponse limitée. Or, cette limitation est indispensable;

- le moment optimum du cycle ostrien auquel l'injection de PMSG doit être effectuée. Ce moment est très important; en effet, l'injection de PMSG pendant la phase lutéale du cycle permet toujours l'ovulation, prolonge parfois la longueur du cycle et conduit souvent à la formation de follicules kystiques (Dowı ING, I949 ; Rowson, I95I ; WILLETT et al., I953). Par contre, l'injection en phase folliculaire 
fournit de meilleurs résultats. A cet effet, on peut donc, soit énucléer le corps jaune (Hammond et Bhattacharya, I944; Brock et Rowson, I952 ; Dowling, I949), soit faire l'injection entre le $I 5^{\mathrm{e}}$ et le $\mathrm{I} 8^{\mathrm{e}}$ jour du cycle.

Nous avons étudié systématiquement l'intervalle entre l'injection de PMSG et l'apparition de l'œstrus suivant, en essayant de faire 1'injection 4 à 5 jours avant cet ostrus. Cependant, par suite de la variabilité de la durée du cycle œestrien chez la Vache (OLDS et SEATH, I95I), nous avons été amenés à utiliser les progestagènes pour essayer de maîtriser l'intervalle injection-œstrus.

Enfin, nous avons cherché à vérifier la répétabilité du traitement retenu.

\section{B. - Infuence des facteurs de variation dépendant de l'animal}

On sait que des facteurs propres à l'animal (âge, race) interviennent dans certaines réponses de l'ovaire aux hormones gonadotropes ou dans la fréquence naturelle des naissances gémellaires (voir plus haut), mais on ignore leurs parts respectives dans la variabilité des réponses de superovulation provoquée. C'est pourquoi dans la plupart de nos expériences, nous avons utilisé des animaux qui avaient la même parité : en général des vaches primipares. En outre, nous avons choisi seulement deux races, parmi les grandes races françaises, dont le taux normal de naissances gémellaires est différent : la race Française Frisonne Pie Noire, race laitière, et la race Charolaise, race à viande.

\section{C. - Fécondabilité des ovocytes superovulés et mortalité embryonnaire}

La proportion d'œufs fécondés après ovulations multiples est faible chez les bovins (KIDDER et al., I952). Si l'injection hormonale est faite en phase lutéale très peu d'ovocytes sont fécondés (WILLETT et al., I952) ; par contre, si l'injection est faite en phase folliculinique, le taux de fécondation est plus élevé (SUGIE et al., I96I). Bien que l'injection de PMSG n'affecte pas le mode de maturation des ovocytes (HAFEZ et IsHrbashi, I964), la présence d'ovocytes anormaux (I5 p. Ioo environ) a été rapportée (HAFEz et al., I963). Il était donc nécessaire de vérifier la fécondabilité des œufs superovulés dans les expériences rapportées ici.

La superovulation entraîne une accélération de la descente des œufs dans l'utérus (Dowl.1NG, I949; HAFEz et al., I963). Ce transport rapide peut provenir d'une élévation importante du taux de progestérone (WINTENBERGER-TORRES, I96I). L'arrivée précoce de l'œuf dans l'utérus peut être une cause de mortalité embryonnaire plus élevée ; il en est de même d'une segmentation plus rapide des oufs superovulés, parfaitement observée chez la brebis (WINTENBERGER-TORRES, I967). L'étude de la mortalité embryonnaire est essentielle dans un programme de naissances multiples. En effet, si la mortalité embryonnaire se produit tardivement, le retour en œestrus des animaux concernés a lieu après des intervalles de temps qui peuvent être longs, parfois de l'ordre de 3-4 mois. Or, on sait déjà que plus le nombre d'ovulations est élevé, plus basse est la capacité de la vache à maintenir une gestation aut-delà de 3-4 mois (GoRdoN et al., I962; HAFEz et al., I965). L'étude des retours en ostrus tardifs après traitement constitue un problème important. 


\section{D. - Inovulation artificielle}

Si la mortalité embryonnaire résulte en partie d'un surpeuplement utérin et si l'on est incapable de maîtriser complètement le taux de superovulation, l'une des solutions au problème des naissances gémellaires consisterait à introduire un cuf fécondé supplémentaire dans l'une des cornes utérines.

On a proposé le nom d’inovulation pour cette opération par analogie avec l'insémination. Nous conserverons ce terme.

Une telle possibilité ne pouvait donc être négligée dans un programme de naissances gémellaires, même si elle ne doit apporter une solution qu'à plus long terme.

Cette méthode présente des avantages certains tels que la possibilité d'induire une gémellité en utilisant toujours les deux cornes utérines : le stock de caroncules est mieux utilisé si l'on a une équipartition des fotus dans les cornes utérines (TEsTART et Du MEsnir, DU Buisson, ig66). Cette méthode permettrait également de surmonter les inconvénients de la variabilité des taux de superovulation. Ennfin, elle diminuerait le nombre d'interventions chez l'éleveur par rapport à la méthode hormonale précédemment décrite, l'inovulation remplaçant l'insémination artificielle.

Mais par sa nature, cette méthode présente deux ordres de difficultés par rapport à la technique hormonale d'induction de la gémellité :

a) C'est d'abord l'obtention, la manipulation, la conservation d'œufs fécondés, pour pouvoir les introduire dans les cornes utérines de vaches, au moment physiologiquement convenable.

Pour transplanter deux œufs fécondés et en voie de développement dans une vache, il convient donc de disposer de tels œufs avec une certaine abondance et de savoir comment on doit les déposer dans les voies génitales de la vache nourrice.

Malgré un aspect assez fondamental, cet ensemble de recherches vise à l'obtention d'une méthode applicable dans la pratique de l'élevage. C'est pourquoi nous avons tenté d'obtenir les œufs à partir d'un matériel abondant et commercial : à cet effet, nous avons décidé de choisir le veau de boucherie femelle comme donneur d'œufs.

Deux voies de recherches ont été conduites simultanément :

- 1'une in vivo, consiste à induire une superovulation (MARDEN, I95I et I953) et à tenter de féconder ces cufs dans les voies génitales du veau, par insémination artificielle, puis à récolter les œufs au stade de développement désiré par abattage de l'animal. Elle n'a pas encore donné lieu à des résultats satisfaisants ;

- 1'autre consiste à opérer intégralement in vitro à partir d'ovaires de veau recueillis à l'abattage, dont des ovocytes sont mis en culture.

On sait que l'ovaire des Mammifères, et celui du Veau en particulier, contient plusieurs dizaines de milliers d'ovocytes bloqués dans un état de repos nucléaire au cours de la prophase méiotique; l'image de ce noyau est caractéristique : c'est le noyau dictyé. On sait également que seuls quelques ovocytes privilégiés, destinés à être ovulés, reprendront l'achèvement de leur maturation nucléaire avant l'ovulation.

Cette maturation consiste en deux divisions successives qui ramènent d'abord le nombre de chromosomes équivalent de $4 n$, soit le double du nombre caractéristique 
de l'espèce pendant toute la période de repos nucléaire, à $2 n$, par l'élimination de $2 n$ chromosomes dans une petite cellule sans importance, le premier globule polaire, puis finalement à $n$, par l'élimination d'un deuxième globule polaire: La formation du premier globule a lieu dans le follicule préovulatoire pendant l'œstrus, puis l'ovocyte est pondu, prêt à émettre le second ; cette émission aura lieu si la fécondation se produit.

Or, Chang (I955), Edwards (I965 $a$ et $b$ ), Susukr et Mastroianni (I966) se sont aperçus que si n'importe quel ovocyte ovarien, ayant achevé sa croissance, était extrait de l'ovaire et placé dans un milieu convenable, il effectuait spontanément cette maturation. EDWARDS et al. (I969) viennent même de montrer que des ovocytes humains, ainsi traités, étaient fécondables in vitro.

C'est cette possibilité de disposer de centaines d'ovocytes à partir d'ovaires de veaux ou de vaches, prélevés aux abattoirs et cultivés pendant une trentaine d'heures qui nous a engagés à poursuivre depuis Ig66 1'étude de la maturation in vitro de l'ovocyte de Veau.

b) Nous avons parallèlement entrepris l'étude des conditions de transfert d'œufs fécondés d'un animal donneur dans l'utérus d'une vache nourrice. Un certain nombre de travaux montrent que la transplantation par voie chirurgicale peut être suivie d'une gestation normale.

Wilitet, Black, Casida, Stone et Buckner (195I), Wili ET, Buckner et LaRSON (I953) furent les premiers à la mettre en évidence en transplantant avec succès I et 3 œufs fécondés. Plus récemment, Rowson, Moor et I,Awson (I969) ont montré qu'en se plaçant dans les meilleures conditions de collecte des cufs et de milieu de culture, le transfert par voie chirurgicale permettait d'obtenir un taux excellent de succès ( $9 \mathrm{I}$ p. cent). Des tentatives faites pour transférer un œuf dans chacune des cornes utérines ont été couronnées de succès (Rowson, communication personnelle).

Mais l'obligation de procéder à une intervention chirurgicale dans la pratique constituait un obstacle important à l'application de cette méthode. Aussi, depuis 20 ans, les transferts par passage direct par le col de l'utérus dans le vagin furent fréquemment tentés sans succès (UMBaUGH, I949; DowlING, I949; Rowson, I95I ; Lamming et Rowson, I952 ; Dziuk et Petersen, I954; Avery et al., I962).

Cependant, Sugre (I965) obtint une naissance par inovulation dans la corne utérine à travers la paroi vaginale en procédant avant la transplantation à une infusion de $\mathrm{CO}_{2}$ dans 1'utérus. Cette technique paraissait résoudre les deux problèmes liés au transfert par voie transvaginale : déclenchement des contractions utérines qui expulsent vers le vagin la morula déposée dans la corne et le risque très élevé d'infection utérine chez la vache pendant la phase lutéale. Cependant, en usant également du gaz carbonique, mais en passant par le canal cervical, Rowson et Moor (I966) obtinrent seulement 3 gestations sur 8 expériences, et Rowson, Moor et Lawson (1969) 4 gestations sur 20 transferts.

L'ensemble des études faites chez la Vache montrait donc que le transfert par voie transvaginale et transcervicale était possible. Il nous a donc semblé permis d'entreprendre avec quelques chances de succès une recherche pour aboutir à une technique offrant les avantages d'un transfert direct dans l'utérus sans prendre les risques d'infection qu'implique la voie transvaginale et en essayant d'éviter une intervention chirurgicale importante impraticable chez l'éleveur. 
c) Enfin, pour pouvoir cultiver les ovocytes immatures, tenter de les féconder après maturation ou pour pratiquer avec une marge de sécurité les transplantations d'œufs d'une femelle dans une autre, il était nécessaire de disposer d'un milieu de culture convenable.

Les études faites nous ont permis de découvrir que le liquide folliculaire convient à la culture in vitro de l'œuf de Vache et permet sa segmentation; jusque-là tous les essais étaient demeurés sans résultat; la qualité spécifique de ce milieu biologique a été confirmée par SREENAN (I968).

Tel est le programme de recherches mis en place pour essayer d'apporter une solution au problème des naissances multiples. En outre, nous avons cherché à prolonger les gestations jusqu'à leur terme afin de pouvoir commencer à étudier les caractéristiques zootechniques des veaux obtenus à partir de naissances multiples.

\section{RÉSUMÉ}

L'une des solutions aux problèmes économiques posés par l'équilibre lait/viande au sein du Marché commun réside dans l'augmentation de la fréquence des naissances gémellaires chez les Bovins. De telles naissances sont normalement observées dans cette espèce. Leur taux d'apparition varie de 0,4 à 4,6 p. Ioo selon les races; faible lors du premier vêlage, il augmente avec l'âge de la vache, au moins jusqu'au huitième ou neuvième vêlage.

Les problèmes zootechniques qui peuvent se poser à propos des naissances gémellaires sont nombreux : rétentions placentaires, fertilité ultérieure des vaches, vitesse de croissance des veaux obtenus, free-martinisme. Cependant, les résultats rapportés sont assez fréquemment contradictoires et, à l'exception de l'existence du free-martinisme, il est difficile de se faire une opinion uniquement d'après la bibliographie.

Aussi, un programme de recherches pour l'induction expérimentale des naissances gémellaires a-t-il été établi, basé sur l'injection d'hormones gonadotropes PMSG et HCG.

Ce programme porte sur:

- la nature, les doses et le moment d'injection des hormones gonadotropes utilisées ;

- l'influence des facteurs de variation des taux d'ovulation dépendant de l'animal ;

- la fécondabilité des ovocytes superovulés;

- la mortalité embryonnaire et la poursuite de la gestation jusqu'à son terme ;

Enfin, des recherches ont été cntreprises sur le transfert d'œufs fécondés, ou inovulation artificielle, autre technique possible pour l'obtention de naissances gémellaires.

\section{SUMMARY}

\section{REASONS FOR OBTAINING TWIN BIRTHS IN CATTIE AND PROCEDURES FOLLOWED}

One of the solutions to the economic problems posed by the milk-meat balance in the Com. mon market is the increase of bovine twin births. Such births are naturally observed in this species, their frequency varying from $0.4 \mathrm{p}$. Ioo to $4.6 \mathrm{p}$. Ioo, depending on the breed. The percentage is low at the first calving, but increases with the age of the cow up to at least the 8 th or 9 th calving.

Animal production problems arising from twin births are numerous. These are : placental retention, subsequent fertility of the cow, growth rate of the calves, and frec-martinism. However, published results are often conflicting and, except for the existence of free-martinism, it is difficult to form an opinion from the bibliography only. 
Therefore, a research program for the experimental induction of twin births has been established, based upon the use of the gonadotrophic hormones PMSG and HCG.

This program deals with :

- the nature, doses, and injection time of the gonadotrophic hormone used;

- the action of ovulation rate variation factors, depending on the animal and the breed ;

- the fertilizability of superovulated oocytes;

- the embryonic survival and development of full-term pregnancy.

Lastly, research was done on the transfer of fertilized eggs, or artificial inovulation, which is another possible method of obtaining twin births.

\section{RÉFÉRENCES BIBLIOGRAPHIQUES}

Anonyme, I966. Twins in cattle. Veetelt-en Zuivelberichten, 9, 377.

Arbeiter K., I962. Vorläufiger Bericht über Versuche zur hormonalen Erzeugung von Zwillingen bei Kühen. Wien. Tieraratl. Mschr, 49, 16I-168.

Arzumanjan E. A., 1965. Multifotation chez les bovins, Dokl. Timiryazev. sel. Khoz. Akad. Mosc., $110,25-30$.

Auriol P., Dumont B. L., Lefebvre J., Duplan J.-M., ig6i. Caractéristiques générales des vaches Charolaises et croissance de leurs produits. Syndicat Contrôle Performances Élevages Nivernais, $28 \mathrm{pp}$.

Avery T. L., Fahning M. L., Pursel V. G., Graham E. F., 1962. Investigation associated with the transplantation of bovine ova. IV. - Transplantation of ova. J. Reprod. Fert., 3, (2) 229-238.

Billère M., 1965. La fécondation en élevage Chavolais. Bull. C.E.T.A., tétude no I032, I-8.

Billère M., I966. Le coût de production du veau Charolais sevré. Bull. C.E.T.A., Étude no IIo8, x9-27.

Boyd H., REed H. C. B., I96r. Investigations into the incidence and causes of infertility in dairy cattle. Fertility variations. Brit. Vet. J., 117, I8-35.

Brands A. F. A., rg66. Enige zootechnische aspecten van retentio secundinarum bij runderen. Thèse Doct. Vet. Sci. Univ. Utrecht, Io7 pp.

Brock H., Rowson L. E., 1952. The production of viable bovine ova. J. Agric. Sci., 42, 479-4.82.

Brodauf H., 1963. Die Zwillingsgeburt des Rindes und ihre Folgen in der Sicht des Rindergesundheitsdienstes. (R. G. D.). Züchtungskunde, 35, 3I6-326.

Casida L. E., Meyer R. K., McShan W. H., Wisnicky W., I943. Effects of pituitary gonadotrophins on the ovaries and induction of superfecundity in cattle. Am. J.Vet. Res., 4, 76-94.

Cloninger W. H., Thoele H. W., I957. Some relationships between twin births and milk production and breeding efficiency. J. Dairy Sci, 40,630.

Cole H. H., Hamburger C., Neimann-Sörensen A., I957. Studies on antigonadotrophins with emphasis on their formation in cattle. Acta Endocr., 26, 286-299.

Comberg G., Velten U., I962. Der Einfluss von Zwillingsgeburten auf Fruchtbarkeit und Gesundheit beim schwarzbunten Niederungsrind. Züchtungskunde, 34, 49-62.

Dabash A. K., 1964. Multiple births relative to milk production and reproduction in the Dutch Friesian breed. Ned. Melken Zuiveltijdschr., 18, $282-284$.

Dowling D. F., I949. Problems of the transplantation of fertilized ova. J. agric. Sci., 39, 374-396.

Dziuk P. J., Donker J. D., Nichols J. R., Petersen W. E., I958. Problems associated with the transfer of ova between cattle. Tech. Bull. Minn. agric. Exp. St., 222.

Dziuk P. J., Petersen W. E., I954. Attempts at non-surgical transfer of bovine ova. J. anim. Sci. 13, roIg.

EDwards R. G., r965 a. Maturation in vitro of human ovarian oocytes. Lancet 2 (74I9), 926-929.

FDWARDS R. G., I 965 b. Maturation in vitro of mouse, sheep, cow, pig, rhesus monkey and human ovarian oocytes. Nature, 208, 349-35r.

Edwards R. G., Bavister B. D., Steptoe P. C., I969. Early stages of fertilization in vitro of human oocytes matured in vitro. Nature, 221, 632-635.

EGLE H., I964. Untersuchungen über die Fruchtbarkeitsverhaltnisse beim deutschen Höhenfleckvieh unter besonderer Beriicksichtigung der Nutzungsdauer und Höhe der Milchleistung : dargestellt anhand der Verhältnisse in der nördlichen Oberpfalz. Abhandlung Dokt. Landw. Fak. Landw. Gartenbau, Tech. Hochsch. Munich, 69 pp.

ERB R. E., Morrison R. A., I959. Effects of twinning on reproductive efficiency in a Holstein-Friesian herd. J. Dairy Sci, 42, 5×2-5 9 .

Foote D. W., Thibault C., ig69. Recherches expérimentales sur la maturation in vitro des ovocytes de Truie et de Veau. Ann. Biol. anim. Bioch. Biophys. 9, 329-349. 
FrEY O., I959. Die Zwillingsträchtigkeit und ihr Einfluss auf Lebenskraft und Milchleistung beim wiuttembergischen Braunvieh. Zïchtungskunde, 31, 55-68.

Gordon I., Williams G., Edwards J., rg62. The use of serum gonadotrophin (PMS) in the induction of twin pregnancy in the cow. J. agric. Sci., 59, 143-198.

HAFEZ E. S. E., Ishrbashi I, I964. Maturation division in bovine oocytes following gonadotrophin injections. Cytogenetics, 3, I67-I83.

Hafez E. S. E., Jainudeen M. R., Lindsay D. R., ig65. Gonadotropin-induced twinning and related phenomena in beef cattle. Acta Endocr., 50, suppl. Ioz, 5-43.

Hafez E. S. E., Rajakoski E., Anderson P. B., lrost O. L., Smith G., r964. Problems of gonadotropin induced multiple pregnancy in beef cattle. Am. J. Vet. Res., 25, 1074-1079.

Hafez E. S. E., Sugie T., Gordon I., r963. Superovulation and related phenomena in the beef cow. I. - Superovulatory responses following PMS and HCG injections. J. Reprod. Fert., 5, 359-379.

Hafez E. S. E., Sugle T., Hunt W. L., I963. Superovulation and related phenomena in the beef cow. Il. - Effect of ostrogen administration on production of ova. J. Reprod. Fert., 5, 38 I-388.

Hammond J. (Jr.), I949. Induced twin ovulation and multiple pregnancy in cattle. J. agric. Sci., 39, $222-225$

Hammond J. (Jr.), Bhattacharya P., 1944. Control of ovulation in the cow. J. agric. Sci., 34, I-I5.

Hewitr A.C.T., 1934. Twinning in cattle. J. Dairy Res., 5, Ior-Io7.

H.ristov V., I966. Croissance et développement des veaux jumeaux (en bulgare). Zhivot. Nauk., 3, $76 \mathrm{I}-768$.

Jainudeen M. R., Hafez E. S. E., Gollnick P. D., Moustafa L. A., ig66. Antigonadotropins in the serum of cows following repeated therapeutic pregnant mare serum injections. Am.J.Vet. Res., $27,669-675$.

Johansson I., I932. The sex ratio and multiple births in cattle. Z. Ziucht. Biol., 24, 183-268.

Johansson I., VENGE O., 195I. Studies on the value of various morphological characters for the diagnosis of monozygocity of cattle twins. Z. Tierzïicht. Zïcht. Biol., 59, 389-424.

Keller K., TANDler J., igr6. Über das Verhalten der Eihäute bei der Zwillingsträchtigkeit des Rindes. Untersuchungen über die Entstehungsursache der Geschlechtlichen Unterentwicklung von weiblichen $Z$ willingskälbern weIche neben einem männlichen Kalbe zur Entwicklung gelangen. Wiener. Tier. Artz. Wochschr., 3, 5I3-526.

Kidder H. E., Barrett G. R., Casida L. E., I952. A study of ovulations in six families of HolsteinFriesians J. Dairy Sci, 35, 436-444.

KRIVINSKII I. 1., I962. The growth of Bestuzhev cattle born in multiple calvings. Uchen. Zap. kazan. vet. Inst., 86, $27 \mathrm{I}-287$.

Labhsetwar A. P., Tyler W. J., Casida L. E., I963. Analysis of variation in some factors affecting multiple ovulations in Holstein cattle. J. Dairy Sci, 46, 840-845.

Lamming G. E., Rowson L. E. A., I952. Superovulation and ovum transplantation in cattle. Proc. IInd int. Congr. Physiol. Path, anim. Reprod, artif. Insem. Copenhagen, 1, I44-I53.

Lillie F. R., I9i6. The theory of the free-martin. Science, 43, 6II-613.

Lillie F. R., IgI7. The free-martin : a study of the action of sex hormones in the fotal life of cattle. J. Exp. Zool., 23, 37I-452.

Lush R. H., 1925. Inheritance of twinning in a herd of Holstein cattle. J. Hered., 16, $273-279$.

Maijala K., I964. Fertility as a breeding problem in artificially bred populations of dairy cattle. I. Registration and heritability of female fertility. Ann. Agric. Fenn., 3, suppl. I, 94 pp.

Martinenko N. A., I965a. Naissances gémellaires chez les vaches de race laitière (en russe). Visn* Sil-hospod. Nauk., 2, 87-89.

Martinenko N. A., r965 b. Naissances gémellaires chez la Vache. Editions Urozhai, Kiev.

Matassino D., Bordi A., I967. The vital statistics of Marche. II. - Calvings, births; age at first service, first conception and calving; calving interval and its fractions. Produz. Anim., 6, 99-II4.

Matassino D., Gargiulo V., Ig65. Vital statistics of Italian Friesian cows. II. - Calving and births. Produz. Anim., 4, 23-7I.

Matassino D., Marati M. A., I964. Live weight at birth of calves of the Marche breed and factors affecting it. Produz. Anim., 3, 263-303.

Meadows C. E., Lush J. L., I957. Twinning in dairy cattle and its relation to production. J. Dairy Sci., 40, I430-1436.

Mechling E. A. II, Carter R. C., I964. Selection for twinning in a grade Aberdeen-Angus herd. J. Hered., 55, 73-75.

Menissier F., I965. Analyse préliminaire du taux de naissances gémellaires dans la zone d'élevage du centre d'insémination artificielle de Naves. St. Génét. anim., C. N. R. Z., 78 - Jouy-en-Josas (France).

Morrison R. A., ERB R. E., 1957. Factors influencing prolificacy of cattle. 1. -- Reproductive capacity and sterility rates. Tech. Bull. agric. Exp. Stn. Wash. St., 25, 1-40.

Nakahara T., Yamauchi M., Kataoka T., Kaneda Y., rg64. Studies on the antihormone against gonadotropin in cattle. Proc. Vth int. Congr. anim. Reprod. artif. Insem., Trento, 3, 338-342. 
Olds D., SEAth D. M., I95I. Repeatability of the estrous cycle length in dairy cattle. J. Dairy Sci., 34, $626-632$.

PARLow A. F., I960. The biological half-life of LH, PMSG and HCr in the rat. Feder. Proc., 19, 155

l'éro R., I968. Dans Contribution de l'I. N. R. A. à l'étude des problínes posés par les rapports entre les productions bovines de lait et de viande. S. E. I., Etude $n^{0} 36, \mathrm{r}-6$.

Petcu D., Calotoru A., I967. Naissances gémellaires chez les bovins laitiers. Lucr. Stiint. Inst. Cerc. Zootech., 25, 363-37r.

PFau K. O., Bartlett J. IV., Sulvart C. E., r948. A study of multiple births in a Holstein-Friesian herd. J. Dairy Sci., 31, $24 \mathrm{I}-254$.

PORIN F., 1968. Quelques aspects de la production de viande bovine en suède. Journées 1i.N.C.E.T.A., Etude $n^{\circ} 1367,1-5$.

RENDEL J., I963. A study of the variation in cattle twins and pairs of single-born animals. I. - Description of the material and the diagnosis of zygosity. Z. Tierzuicht. Ziichtbiol., 79, 75-85.

RichteR F., I955. Untersuchungen über den Verbleib der Nachkonmen von R. L. Kühen des Höhenviehs. Z. Tierziicht. Ziicht. Biol., 65, 223-242.

Rowson L. E. A., I95I. Methods of inducing multiple ovulation in cattle. J. Endocr., 7, 260-270.

Rowson L. E. A., Moor R. M., I966. Non-surgical transfer of cow eggs. J. Reprod. Fert., 11, 3II312.

Rowson L. F. A., Moor R. M., Lawson R. A. S., I969. Fertility following egg transfer in the cow ; effect of method, medium and synchronization of cestrus. J. Reprod. Fert, 18, 517-523.

Schilling E., Holм W., r963. Experimentelle Untersuchungen zur hormoninduzierten Zwillingstriichtigkeit beim Rind. Z. Zuchthyg. Fortpl. Besam., 7, I79-204.

Schultze A. B., rg66. Do we want twins? A. I. Dig., 14, 6-I2.

SREevan J., I968. In vivo and in vitro culture of cattle eggs. VI $t$. Cong. intern. Reprod. anim. Insem. artif., 1, 577-580.

Sugre T., 1965. Successful transfer of a fertilized bovine egg by non-surgical techniques. J. Reprod. Fert., 10, I97-201.

Sugie T., Hunt W. L., I96I. Experimental bovine superovulation following suppression of ovaries with estrogen. J. anim. Sci., 20, 979-980.

Testart J., du Mesnil du Buisson F., rg66. Fitude biométrique des placentomes dans les gestations simples ou gémellaires des bovins. Ann. Biol. anim. Bioch. Biophys., 6, 483-493.

Thibault C., 1966. La culture in vitro de l'ouf de vache. Ann. Biol. anim. Bioch. Biophys., 6, 159I64.

Turman E. J., Renberger R. E., Stiphens D. F., ig68. Multiple births in beef cows treated with PMSG. 60th Annual Meeting American Society of Animal Sciences, Stillwater, Oklahoma, I 2 pp.

Turman E. J., Renberger R. E., Stephens I). F., ig69. Producing multiple births in beef cows by hormone injections.

Umbavgh R. E., I949. Superovulation and ovum transfer in cattle. Am. J. Vet. Res., 10, $295-305$.

Vandenbusscine O, Vandenbussche P., Vandeplassche M, I964. Dystocia in the Red cattle of West Flanders. Vlaams diergeneesk. Tijdschr., 33, 205-215.

VISsac B., Communication personmelle.

IVillett E, L., Black W. G., Casiva I. E., Stonf, W. H., Buckner P. J., I95i. Successfull transplantation of a fertilized bovine ovum. Science, 113, 247.

Willett E. L., BUCKNiR P. J., LARSON G. L., 1953. Three successfull transplantations of fertilized bovine eggs. J. Dairy Sci., 36, 520-523.

Willett E. L., Buckner P. J., McShan W. H., 1953. Refractoriness of cows repeatedly superovulated with gonadotropins. J. Dairy Sci, 36, 1083-1088.

Willett E. L., MCShan W. H., Meyer R. K., 1952. Relation of stage of cycle and source of luteinizing hormone to super-ovulation in dairy cattle, Proc. exp. Soc. Biol. Med., 79, 396-400.

Wintenberger-Torres S., I96I. Mouvement des trompes et progression des œufs chez la Brebis. Ann. Biol. anim. Bioch. Biophys., 1, I2I-I33.

WINTEMBERGER-Torres S., I967. Étude expérimentale de la migration tubaire des aufs et de leur segmentation chez la Brebis. Thèse, Paris, Io8 pp.

Zavanowski M. M., Eskin I. A., r939. L'utilisation du prolan et du sérum de jument gravide pour le contrôle du cycle sexuel chez la vache. Trud. Dinam. Razvit., 11, II2-132. 